\title{
A Connected Chair as Part of a Smart Home Environment
}

\author{
Marc Hesse*, André Frank Krause ${ }^{\dagger}$, Ludwig Vogel ${ }^{\dagger}$, \\ Bhavin Chamadiya $^{ \pm}$, Michael Schilling ${ }^{ \pm}$, Thomas Schack ${ }^{\dagger}$ and Thorsten Jungeblut*
}

\begin{abstract}
The connected chair is part of the Supportive Personal Coach in the KogniHome project, which offers guided fitness training, relaxation, and assistive functions. The chair comes with integrated sensors, actuators, control logic and wireless transceiver. The sensors are able to measure respiration and heart rate as well as the user's actions. The actuators are used to adjust the chair to the actual user's needs and the transceiver is used to connect wireless sensor nodes and to exchange data with a base station. Additional value is generated by connecting the chair to the smart home environment, which enables and expands novel features and applications.
\end{abstract}

\section{INTRODUCTION}

The development of the described connected chair is part of the KogniHome [1] project, which is divided in three main sub-projects: Kitchen, Entrance and Supportive Personal Coach. In the KogniHome project, partners from industry, research, service industries as well as social services and health providers address the question of how 'intelligent' and 'trustworthy' technical systems can be realized to help people in their everyday lives. The chair is part of the Supportive Personal Coach sub-project, which is a ubiquitous interaction system with the goal to improve the user's well-being. This includes qualified fitness training, a relaxation mode and assistive functions. To realize these functions, several sensors, actuators, control logic and wireless interface were integrated into the chair. The secondary goal is to seamlessly integrate the whole system into the living room, hence every component was integrated into the chair.

The developed chair is based on a series-production reclining armchair with actuated seating, footrest and backrest. During the development, an additional actuation of the headrest was added. Furthermore, several force sensitive resistors (FSR), a galvanic skin response sensor (GSR) and a radar sensor for contact-less measurement of the heart and respiration rate were integrated. A microcontroller reads out the sensors, controls the actuators and communicates wirelessly with a base station and additional wearable sensors via Bluetooth Low Energy (BLE). The developed connected chair (KogniChair) is displayed in figure 1.

\footnotetext{
*Authors are with the Cognitronics and Sensor Systems Group, Cluster of Excellence Cognitive Interaction Technology (CITEC) at Bielefeld University, 33619 Bielefeld, Germany mhessedcit-ec.uni-bielefeld.de

${ }^{\dagger}$ Authors are with the Neurocognition and Action - Biomechanics Group, Cluster of Excellence Cognitive Interaction Technology (CITEC) at Bielefeld University, 33619 Bielefeld, Germany

${ }^{ \pm}$Authors are with Advanced Engineering Group at Hella KGaA Hueck \& Co., 59552 Lippstadt, Germany michael.schilling@hella.com
}

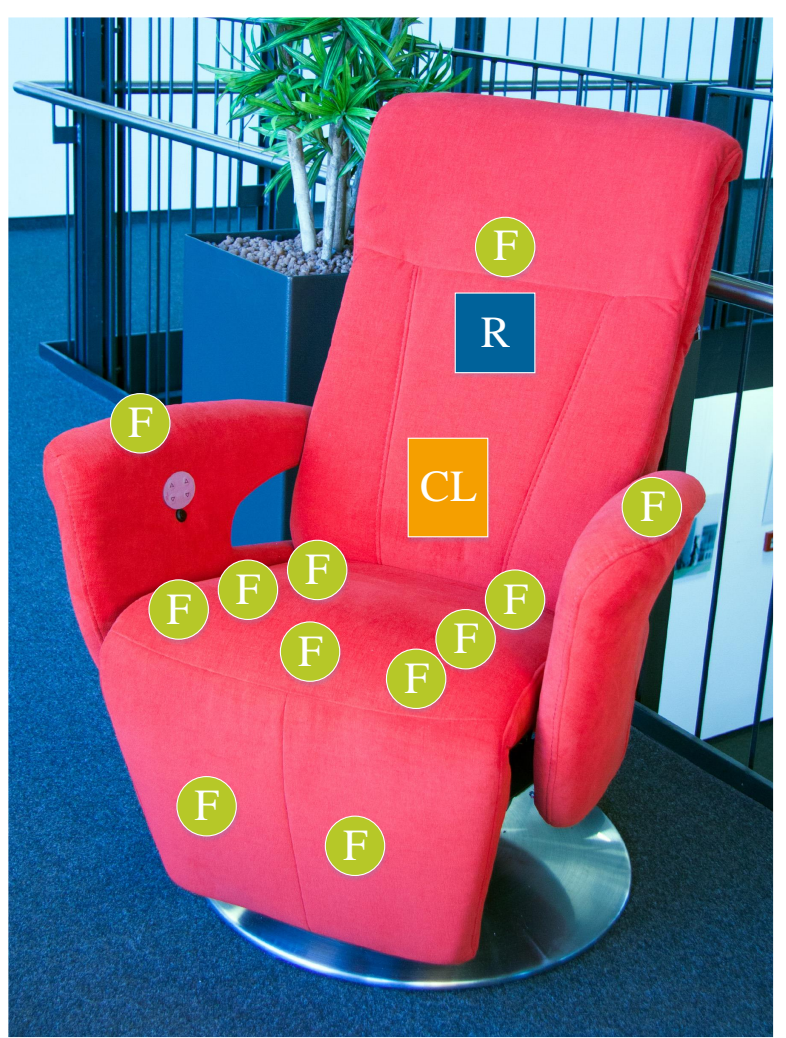

Fig. 1. The KogniChair with integrated force sensitive resistors (F), radar sensor (R), actuators, and control logic (CL)

The involved industrial partners Hettich [2] and Hella were responsible for the mechanical base including actuators and the development of the radar sensor, respectively.

This paper is structured as follows: Section II summarizes related work, before we introduce our system architecture in section III. Basic applications are described in section IV. Afterwards, the concept of the Supportive Personal Coach is presented in section V. Finally, the paper is concluded in section VI and a prospect on our future work is given in section VII.

\section{RELATED WORK}

One important aspect of a connected chair is non-obtrusive and contact-less cardiorespiratory monitoring [3]. Several approaches to integrate such technologies into items of daily life can be found in literatures. Often capacitively coupled electrocardiography (CCECG) is utilized, which enables the contact-less measurement of an ECG. It has been integrated in an office chair [4] as well as in a wheelchair, a hospital 
bed [5] and a vehicle [6]. Furthermore, several techniques are combined to acquire parameters, which are not directly measurable. Contact based ECG and photoplethysmograhy (PPG) were combined to determine the blood pressure based on the pulse arrival time by $\mathrm{Wu}$ et al. [7]. Baek et al. [8] integrated ECG, PPG and ballistocardiography (BCG) sensors into a chair to acquire heart rate and blood pressure. Teichmann et al. are working on a combination of optical coupling (PPG) and electromagnetic coupling, which is used to capture heart rate and respiration rate [9]. This concept, together with a mechanical coupling (BCG) has recently been integrated into a dental chair [10] and seems to be a promising approach. In addition to cardiorespiratory monitoring, often the user's behavior and movements are tracked, which can be done by integrating pressure or force sensors. Martins et al. [11] integrated matrices of 2-by-2 pressure cells into the seating and the backrest of an office chair to classify the user's sitting posture. Eight FSRs were integrated into a lay-on for office chairs by Hermann and Kõiva [12] and used to detect and sonificate the user's posture. A similar work to ours was showcased with the GewoS chair [13]. Health parameters like blood pressure, oxygen level and ECG were measured and visualized on a remotely connected TV. The users' actions were monitored with integrated strain gauges. Furthermore, the sensors' data was used to monitor fitness exercises for elderly people in their own homes. But the exercises were rather demanding (e.g. rowing) and the actuators were not seamlessly integrated.

\section{SYSTEM ARCHITECTURE}

The hardware requirements for the control logic are: reading the sensor values, controlling the actuators and communicating with other systems in the smart home environment of the KogniHome project. The block diagram of the KogniChair control logic is shown in figure 2. It is based on a SAM4L Xplained Pro evaluation board from Atmel, which includes the ATSAM4LC4C microcontroller together with an embedded debugger (Atmel $E D B G$ ). To interface and control every part of the chair, several additional electronic components were necessary. Therefore, a printed circuit board was custom designed and attached to the evaluation board.

In total, 12 FSRs from Interlink Electronics are integrated into the chair's fabric (fig. 1). In detail, seven are integrated in the seating (FSR406), two in the footrest (FSR406), one in each armrest (FSR408) and one in the backrest (FSR406). Each of the FSRs forms a voltage divider with a digital potentiometer ( 2 x Analog Devices AD5206 with 6 channels each). This enables to vary the resistance and consequently the FSR sensitivities during run time for different chair requirements. The output voltages are acquired using an ADC (Texas Instruments ADS7953) with 16 channels. Further, the GSR sensor's signal is amplified by a Microchip MCP6241T operational amplifier. Subsequently, it was filtered and acquired by the ADC. The remaining 3 channels are used to measure the current of the three actuators, which are outputs from the motor drivers $(3 \mathrm{x}$ Texas Instruments

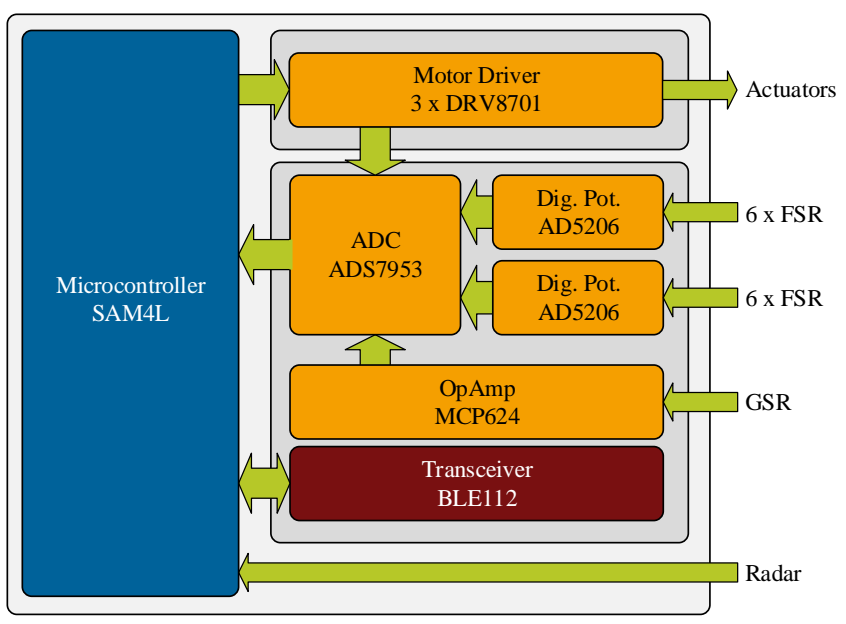

Fig. 2. Block diagram of the KogniChair control logic

DRV8701). The drivers are controlled by the microcontroller via PWM. The wireless interface consists of a Bluetooth Low Energy transceiver (Bluegiga BLE112). The radar sensor is integrated into the backrest (fig. 1) and directly connected to the evaluation board using a serial interface. The control logic is mounted in a housing (fig. 3) and fully integrated into the chair's backrest. Because power is supplied by an internal battery pack, the chair is mobile and not fixed to a specific location.

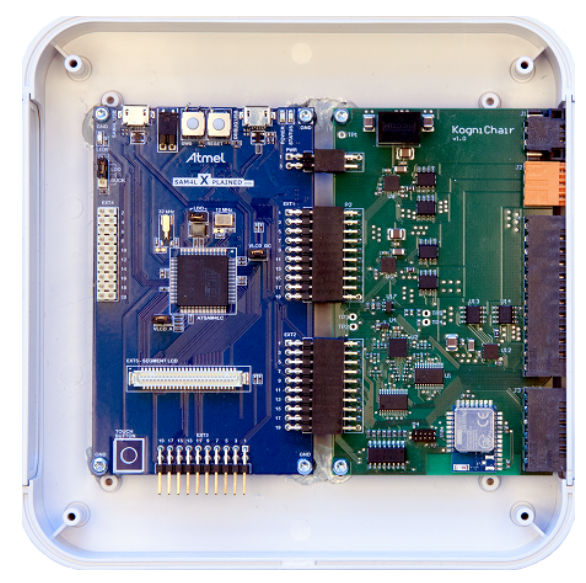

Fig. 3. KogniChair control logic including evaluation board, custom printed circuit board and housing

The BLE interface is used for communication with the base station (sec. V). The data transmission includes sensor data, status signals and control commands. Thus, the chair is integrated into the smart home environment and can be adapted to the user's needs. Furthermore, the wireless interface allows the integration of wireless body sensors. Due to the implementation of the standardized Heart Rate Profile [14], a large variety of heart rate monitors is supported. In our case, the MIO Link heart rate monitor and the $L G$ Urbane smart watch are used. Additionally, custom services for our own sensor nodes can be implemented. 


\section{BASIC APPLICATIONS}

The integrated sensors and actuator enable a multiplicity of applications. For instance the FSRs in the seating can be used for presence detection. If a user sits down, the information can be transmitted to the smart home and nearby services like lightning and heating can be adapted to the user. Furthermore, it is possible to determine the user's posture by analyzing patterns in the sensor data, which can be used to emphasize a healthy sitting posture. Additionally, the FSRs can serve as simple push buttons and form an input device, e.g. switching TV channels by tapping on the armrests, controlling computer games and control of the chair itself.

The continuous wave radar sensor measures the movements of the body caused by the beating of the heart and the respiration. Example of the raw radar signal is shown that is used to calculate the respiration rate (fig. 4) and heart rate (fig. 5). With these parameters, that are acquired contactless, it is possible to track the user's health status, detect potentially harmful conditions and provide biofeedback for the relaxation modes (e.g. deep breathing for guided meditation).

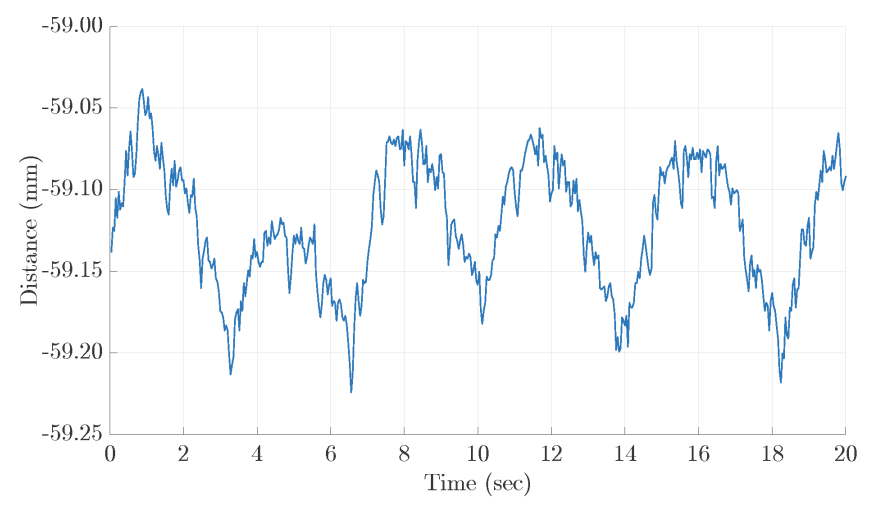

Fig. 4. Raw signal of the radar sensor. The inhalation and exhalation of

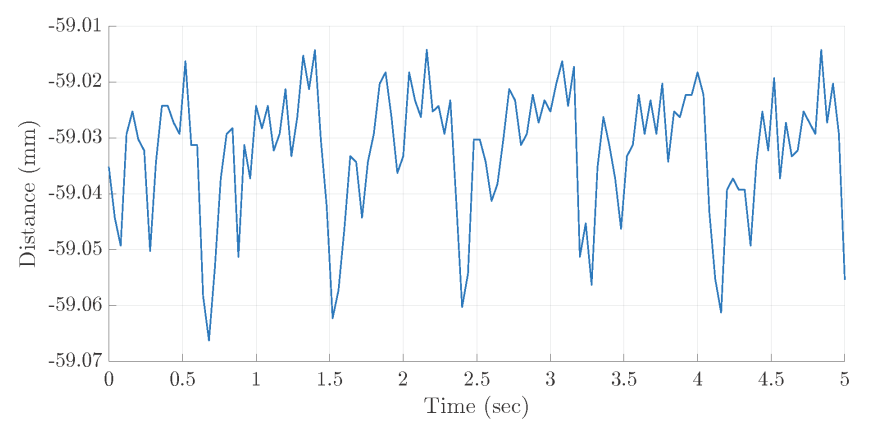

Fig. 5. The distance signal of the radar sensor shows a QRS like signal that is used to determine the heart rate

The connected wearable is used to identify the user. The receiver checks the received MAC address of the sender and fetches the user preferences from a database. Furthermore, it is possible to estimate the distance between the chair and the user by evaluating the received signal strength (RSSI). If the connected wearable has a heart rate sensor, the heart rate can be used in case the radar sensor's signal is disturbed or

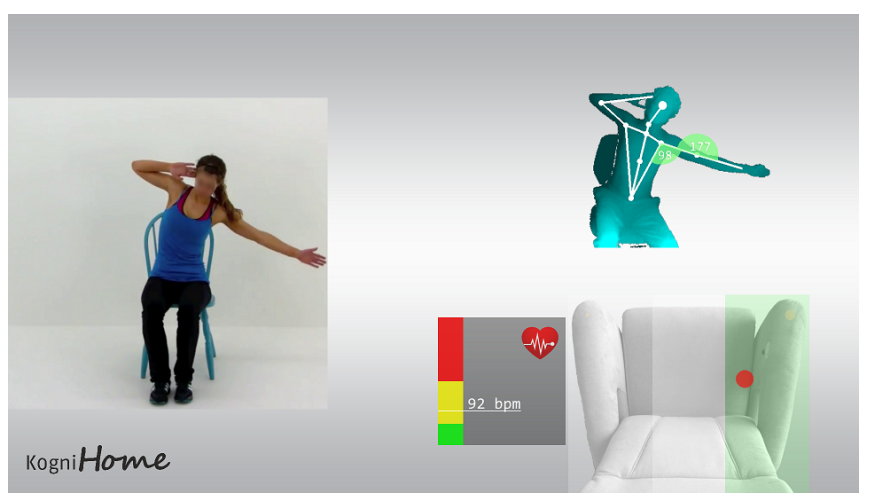

Fig. 6. Screen-shot of a Personal Coach exercise with instructional video, real time feedback of the motion tracking, user posture on the chair and current heart rate

in case the user doesn't sit in the chair. The fusion of both sensors potentially increases reliability and robustness.

The actuators are used to assist the user while sitting down or standing up, which is especially important for elderly people. The seating is raised, once the user is identified and approaches the chair. As soon as the user sits down, which is detected by the FSRs, the chair moves into the preferred position. If the user wants to stand up, the seating is raised and stays in position until the user stood up. Finally, the chair returns to its initial position. During each adjustment of the chair, the FSRs form a feedback loop with the user. Moreover, the actuator can be used to adjust the chair to the actual user's need. For example, establishing a relaxing position while watching a movie or a more upright position during reading. Information about the individual needs are based on the identification of the user and can be gathered from the connected database. Therefore, the chair can be adjusted for each individual member of the family and can react to, for instance, a person's physical condition and the time of the day.

To grant the user's autonomy, every automatism can be overruled by the user and the chair can be manually controlled with additionally integrated push buttons.

\section{SUPPORTIVE PERSONAL COACH}

The described KogniChair is part of the Supportive Personal Coach (SPC), which is designed to be an ubiquitous interaction system: by combining and enhancing state-of-the art methods mental diagnostics, marker-free motion tracking and natural interaction, the system will seamlessly integrate into the living room and provide a motivating and fun exercise tool, a relaxation mode and entertainment through gamified activities. The personal coach base-station is a Intel NUC6i5SYH, which is connected to a TV. It fuses all data streams and, together with marker-free motion tracking, provides a closed-loop sensorimotor learning, interaction and training system. Neurocognitive diagnostic tools [15] enable the SPC to identify user specific problems in the execution of action sequences based on measured memory structures. 
To guide the user to the appropriate exercise, the system integrates adaptive training schedules, training history, daily activity data and memory structures. This status diagnostic enables the system to adjust the exercise selection for every user and considers the fitness level, the personal requirements and the physical and mental state on the day. Keeping in line with the dynamic assessment approach [16] the training changes are used to measure the learning and action potential. Based on this diagnostic, the user profits from the guidance of the system by practicing in his zone of proximal development. Marker-free motion tracking and gesture based interaction with the interface is implemented using a depth camera (Microsoft Kinect 2). The usability of the interaction model was optimized for elderly people and improves the default Kinect model. Providing real-time postural feedback using the Kinect skeletal tracking (fig. 6) enables a closedloop sensorimotor learning process: a training session with a complex movement sequence is separated into distinct movement phases according to pre-identified key-frame postures. The next movement sequence starts only after a correct posture is held by the user for a predefined amount of time. This timespan adapts to the skill of the user. For a skilled user the waiting time reduces to zero, allowing for a smooth training session. For novices, postural feedback is given via text and speech, if the key-frame posture is not attained within a certain time limit.

Daily activity data is based on the measured respiration and heart rate and the users' actions, which includes the motion and posture feedback from the FSRs and the Kinect tracking. Additionally, the data collected by the wearables (e.g. walked distance) is combined with the SPC's daily activity data to estimate the user's energy expenditure [17] during the day. These values could be used to select recipes in the sub-project Kitchen [18]. The GSR sensor will be used in the relaxation mode and is part of a biofeedback loop.

\section{CONCLUSION}

We presented an intelligent and connected chair with integrated FSRs, GSR sensor, radar sensor and actuated seating, footrest, backrest and headrest. Its sensors and actuators are controlled by a microcontroller based control logic and its data can be transmitted wirelessly. Every component is seamlessly integrated so that the chair looks like a seriesproduction reclining armchair. Therefore, it blends in the living room and has the potential to generate a high acceptance rate among users. The introduced applications are potentially able the improve the user's well-being by offering qualified fitness training, a relaxation mode and assistive functions. In contrast to previous work, additional value is generated by connecting the chair to a smart home environment, which enables and expands novel features and applications.

\section{FUTURE WORK}

In future work, we want to extend the applications by connecting SPC and KogniChair with more parts of the smart home. In the near future, every component of the KogniHome project will be integrated into an apartment, which will serve as a demonstrator. This enables us to conduct user studies in which we will validate the described functionality and quantify user acceptance. In future publications, the SPC will be described in detail and the outcome of the user studies will be presented.

\section{ACKNOWLEDGMENT}

This research was funded by grants from the Cluster of Excellence Cognitive Interaction Technology 'CITEC' (EXC 277), Bielefeld University and the German Federal Ministry of Education and Research (BMBF) within the project 'KogniHome'. The authors are responsible for the contents of this publication.

\section{REFERENCES}

[1] [Online]. Available: https://www.kogni-home.de/

[2] [Online]. Available: https://www.hettich.com/en/home.html

[3] C. Brüser, C. H. Antink, T. Wartzek, M. Walter, and S. Leonhardt, "Ambient and unobtrusive cardiorespiratory monitoring techniques," IEEE Reviews in Biomedical Engineering, vol. 8, pp. 30-43, 2015.

[4] A. Aleksandrowicz and S. Leonhardt, "Wireless and non-contact ecg measurement system - the aachen smartchair," Acta Polytechnica, vol. 47, no. 4-5, 2007.

[5] B. Chamadiya, K. Mankodiya, M. Wagner, R. B. Nasreddine, and U. G. Hofmann, "Non-contact, non-obtrusive electrocardiography in clinical environements," in PervasiveHealth, May 2011, pp. 101-106.

[6] B. Chamadiya, S. Heuer, M. Wagner, and U. G. Hofmann, "Textile capacitive electrocardiography for an automotive environment." in BIODEVICES, 2011, pp. 422-425.

[7] K. f. Wu, C. h. Chan, and Y. t. Zhang, "Contactless and cuffless monitoring of blood pressure on a chair using e-textile materials," in 2006 3rd IEEE/EMBS International Summer School on Medical Devices and Biosensors, Sept 2006, pp. 98-100.

[8] H. J. Baek, G. S. Chung, K. K. Kim, and K. S. Park, "A smart health monitoring chair for nonintrusive measurement of biological signals," IEEE Transactions on Information Technology in Biomedicine, vol. 16, no. 1, pp. 150-158, Jan 2012.

[9] D. Teichmann, D. D. Matteis, T. Bartelt, M. Walter, and S. Leonhardt, "A bendable and wearable cardiorespiratory monitoring device fusing two noncontact sensor principles," IEEE Journal of Biomedical and Health Informatics, vol. 19, no. 3, pp. 784-793, May 2015.

[10] D. Teichmann, M. Teichmann, P. Weitz, S. Wolfart, S. Leonhardt, and M. Walter, "Sensindent - noncontact sensors integrated into dental treatment units," IEEE Transactions on Biomedical Circuits and Systems, vol. PP, no. 99, pp. 1-9, 2016.

[11] L. Martins, R. Lucena, J. Belo, R. Almeida, C. Quaresma, A. P. Jesus, and P. Vieira, Intelligent Chair Sensor - Classification and Correction of Sitting Posture. Cham: Springer International Publishing, 2014, pp. 1489-1492.

[12] T. Hermann and R. Kõiva, tacTiles for Ambient Intelligence and Interactive Sonification. Berlin, Heidelberg: Springer Berlin Heidelberg, 2008, pp. 91-101.

[13] L. Roalter, T. Linner, A. Möller, S. Diewald, and M. Kranz, "The healthcare and motivation seat - a survey with the gewos chair." in Mensch \& Computer Workshopband. Citeseer, 2012, pp. 37-43.

[14] [Online]. Available: https://www.bluetooth.com/specifications/adoptedspecifications

[15] C. Seegelke and T. Schack, "Cognitive representation of human action: Theory, applications, and perspectives," Frontiers in Public Health, vol. 4, 2016.

[16] T. Schack, "Dynamic assessment in sport," Measurement in sport and exercise psychology, pp. 91-101, 2012.

[17] T. Hörmann, P. Christ, M. Hesse, and U. Rückert, "Robust estimation of physical activity by adaptively fusing multiple parameters," in 2015 IEEE 12th International Conference on Wearable and Implantable Body Sensor Networks (BSN), June 2015, pp. 1-6.

[18] A. Neumann, C. Elbrechter, N. Pfeiffer-Leßmann, R. Kõiva, B. Carlmeyer, S. Rüther, M. Schade, A. Ückermann, S. Wachsmuth, and H. J. Ritter, “"kognichef”: A cognitive cooking assistant," $K I$ Künstliche Intelligenz, pp. 1-9, 2017. 